\title{
OPTIMIZING CARBON NANOTUBE CONTACTS FOR USE IN ORGANIC PHOTOVOLTAICS
}

Teresa Barnes, Jeff Blackburn, Robert Tenent, Anthony Morfa, Mike Heben, and Tim Coutts PVSC 2008 $5 / 13 / 2008$ 


\section{Transparent Contacts in PV}

- Thin Film Devices

- CdTe (16.5\%) - TC sees $600^{\circ} \mathrm{C}$

- CIGS (20\%) - TC must be grown at room $\mathrm{T}$

- TCOs

- ZnO:Al, $\mathrm{SnO}_{2}: \mathrm{F}, \mathrm{In}_{2} \mathrm{O}_{3}: \mathrm{Sn}$, $\mathrm{Cd}_{2} \mathrm{SnO}_{4}$

- TCs must meet different requirements in each technology

- Successful demonstration of SWNT electrodes in thin film devices

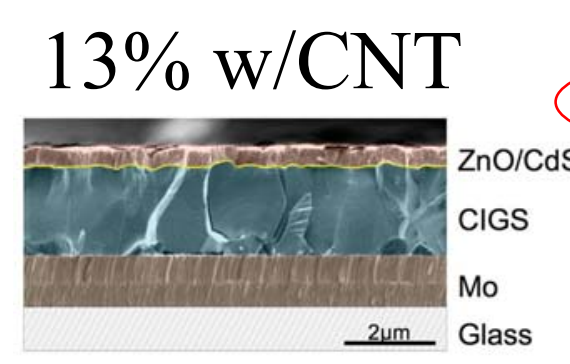

$12 \% \mathrm{w} / \mathrm{CNT}$

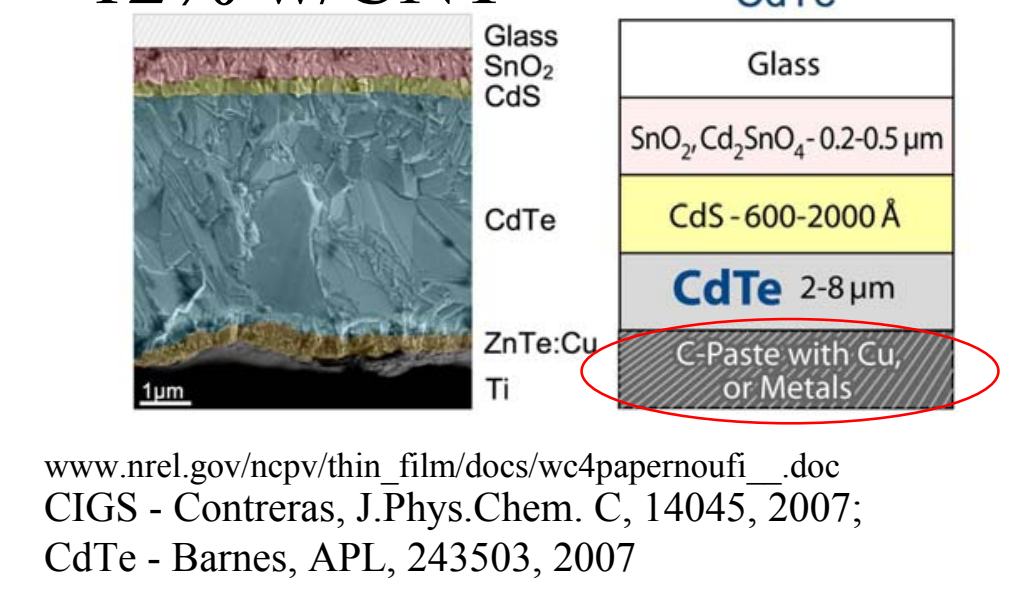

www.nrel.gov/ncpv/thin_film/docs/wc4papernoufi_.doc CIGS - Contreras, J.Phys.Chem. C, 14045, 2007; CdTe - Barnes, APL, 243503, 2007

CIGS

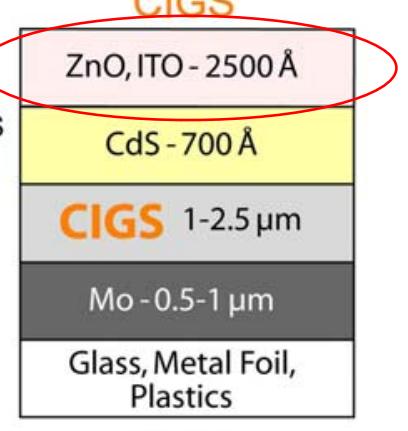

CdTe 


\section{Carbon Nanotube Basics}

Multi-wall tubes

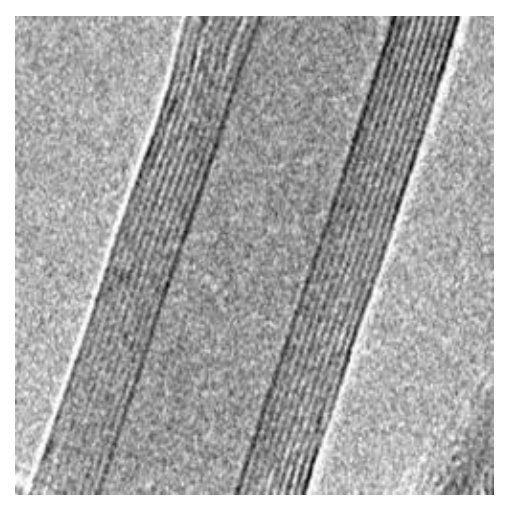

Baughman, Science 2872002

Armchair (m)

Zig-zag

Chiral
Single-wall bundle

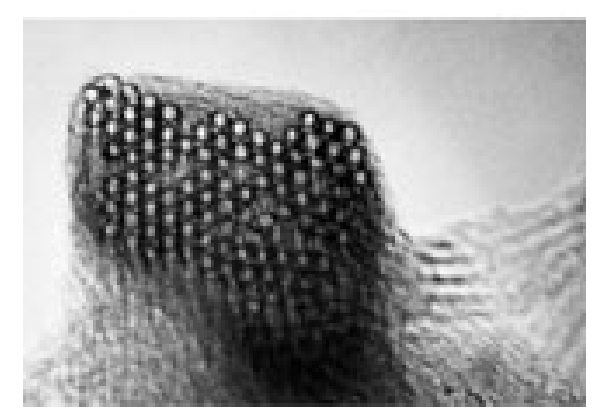

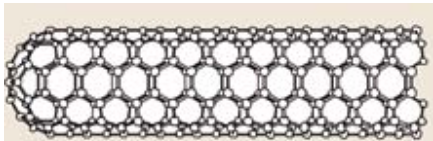
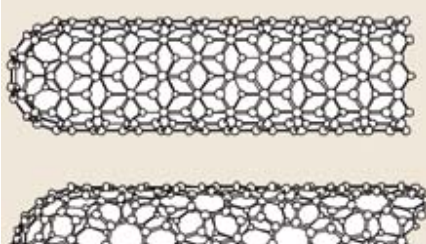

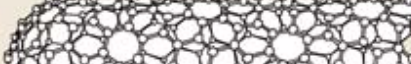

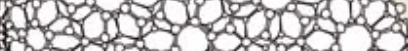

- Many synthesis methods

- CVD, Arc, HipCO, laser vaporization

- Extensive postprocessing

- Impurities

- Multi vs. Single Wall

- Random chirality distribution

$-1 / 3$ metallic, $2 / 3$ semiconducting 


\section{OPV Device Structures Bulk Heterojunctions}
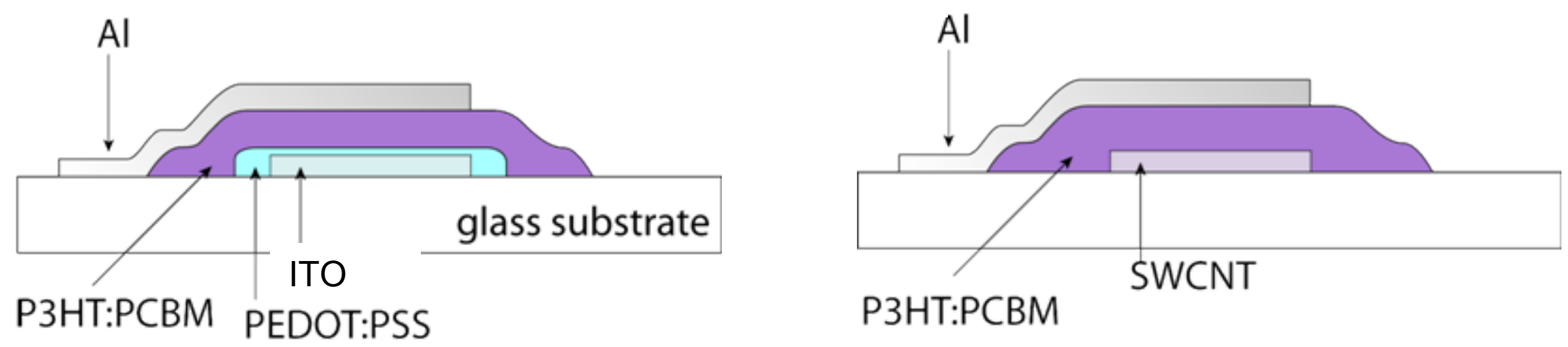

Short diffusions lengths would require impractically thin absorber layers in a "stacked" heterojunction (BHJ 300nm thick)

Mix P3HT and PCBM in a 1:1 ratio to create a "bulk" heterojunction P3HT carries holes to the ITO side, PCBM transports electrons to Al

OPV Device Results:

J. van de Lagemaat, T. M. Barnes, G. Rumbles et al., Applied Physics Letters 88 (23), 3 (2006). 


\section{Early SWCNT Production at NREL}

- All "TCO" work so far uses laser tubes

- $1 \mathrm{~nm}<\mathrm{d}<1.4 \mathrm{~nm}$; all SWCNT

- PLV tubes are very long with low defect density (Raman D/G 1/190)

- Films formed by vacuum filtration/ membrane transfer
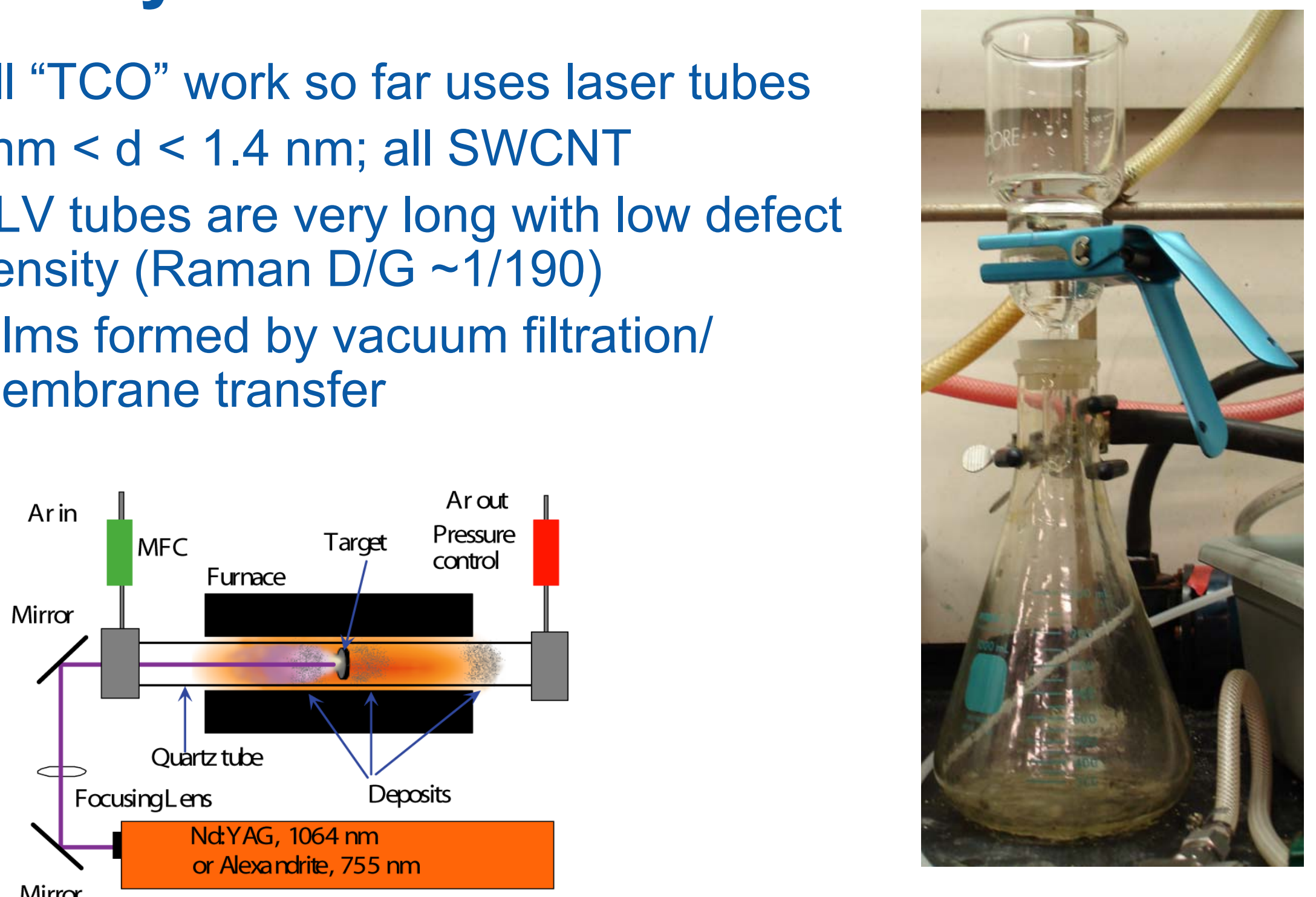

NREL Pulsed Laser Vaporization

Wu, Science. 305, 1274 (2004)

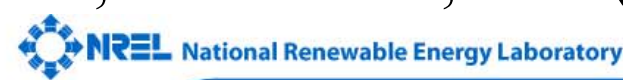




\section{Scalable Production: Ultra-sonic spray}
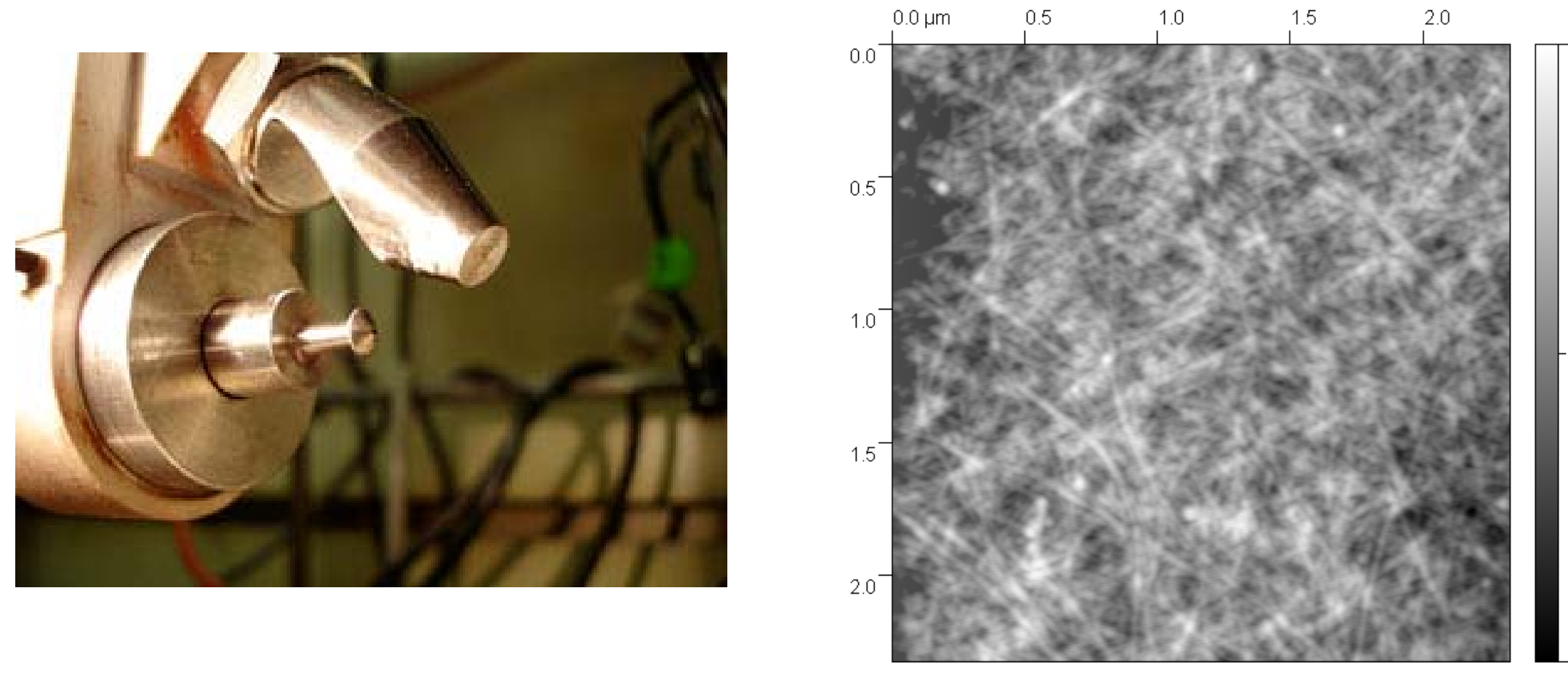

Metrics: Transparency, Conductivity, Stability

Parameter Matrix

Purification: metals, amorphous C

Surfactant: SWCNT dispersion, bundle size Sonication: SWCNT length, bundle size, defects Post Processing: surfactant removal, doping

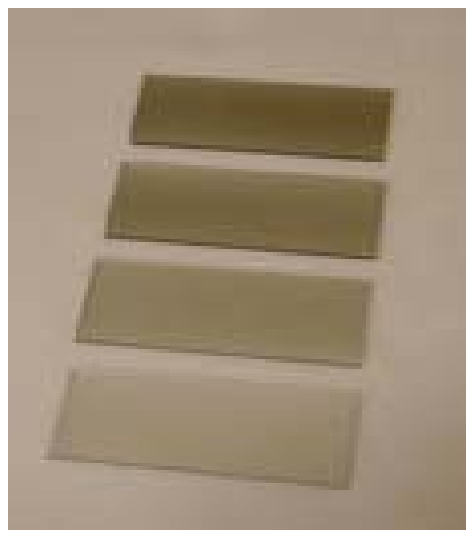

$1 \times 4$ inch 


\section{Ink Formulation for Ultrasonic Spray}

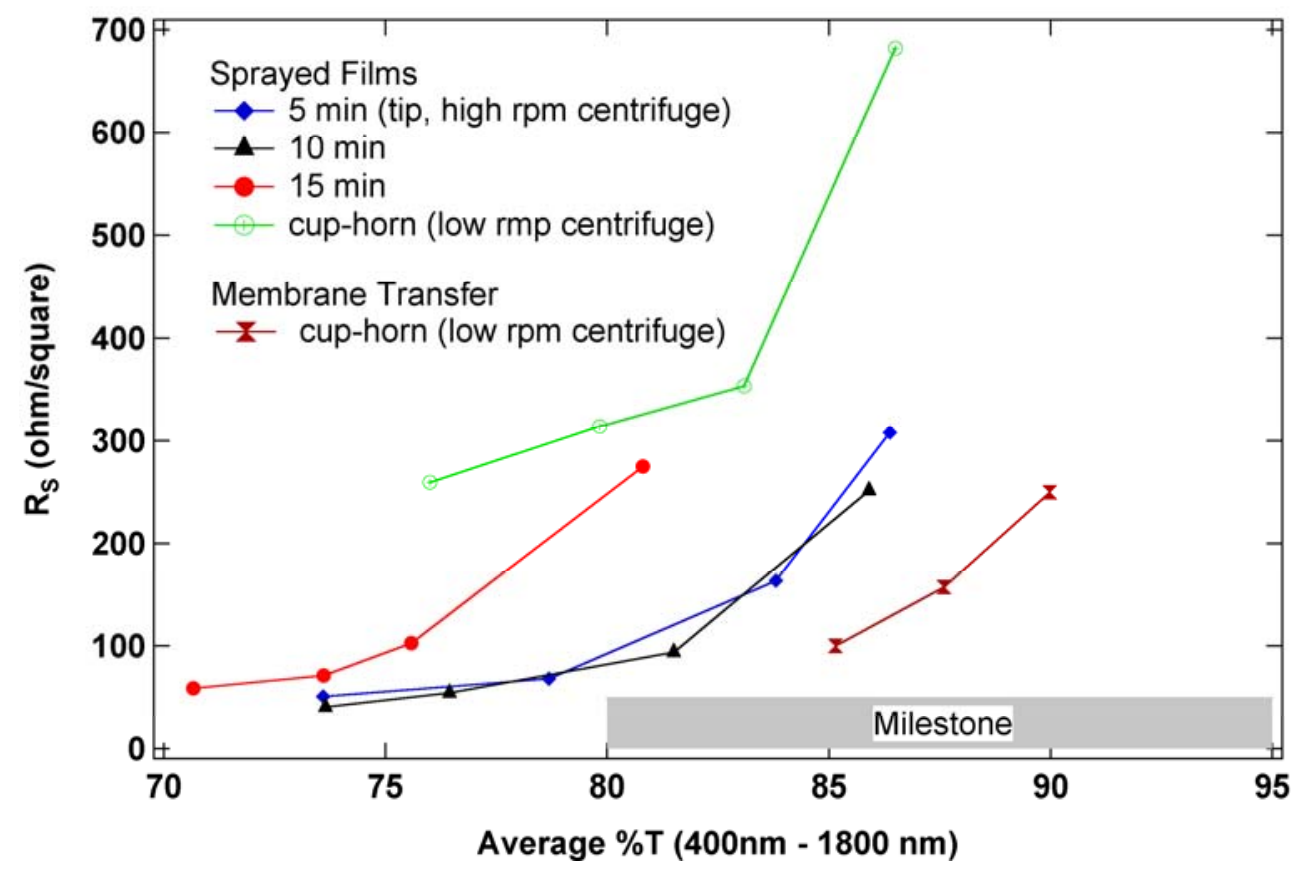

Best
$\mathrm{Bad}$

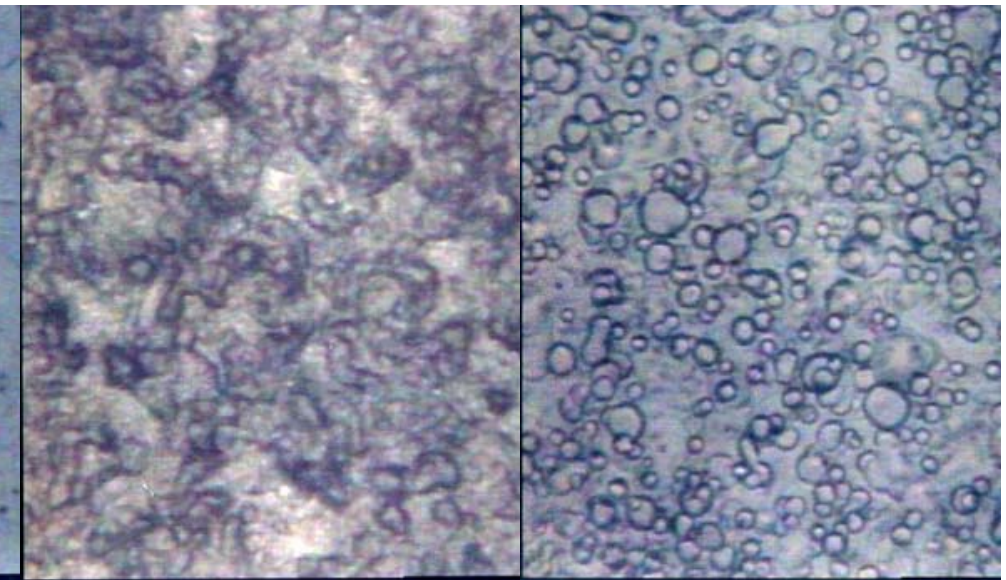

- Aqueous inks

- Ink sonication

- Tube purification/ starting material

- Surfactant choice is key

- Morphology

- Conductivity

- Removal 


\section{Sprayed vs. Membrane Transferred}
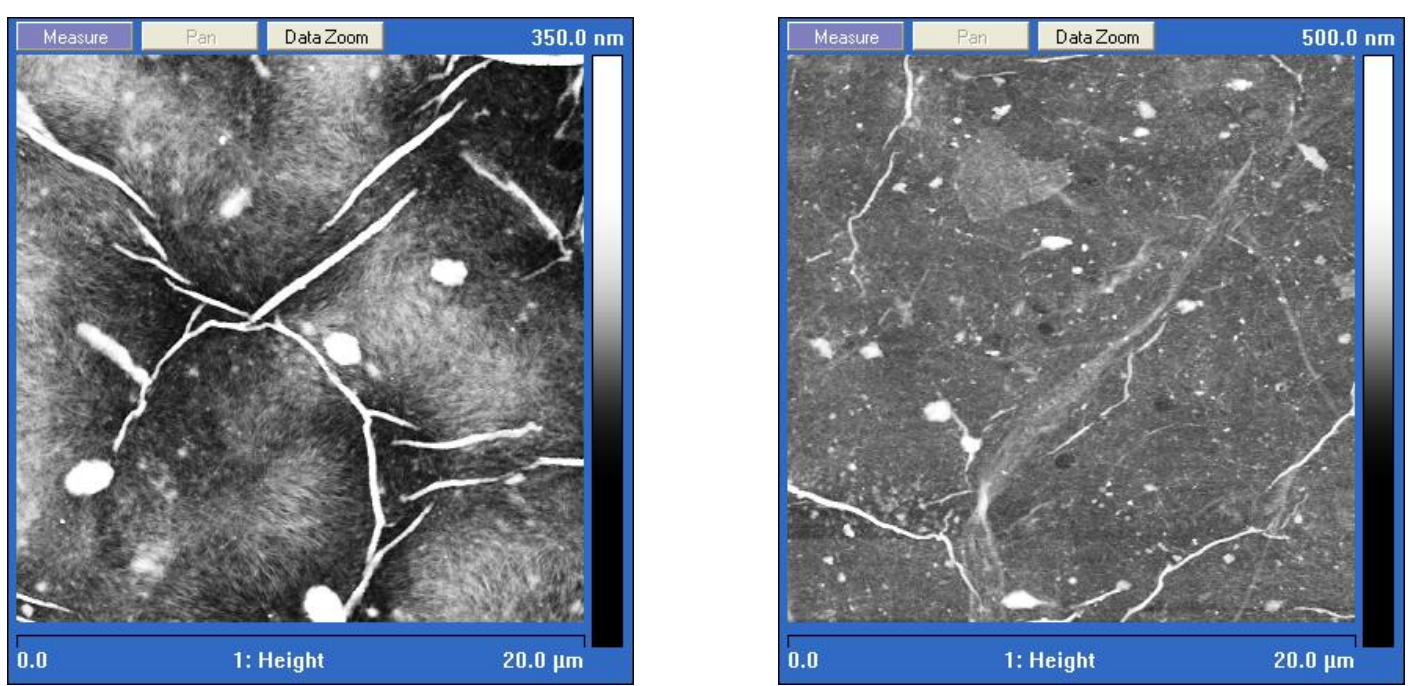

- Large scale roughness in membrane films

- Possible texturing from membrane

- Wrinkling

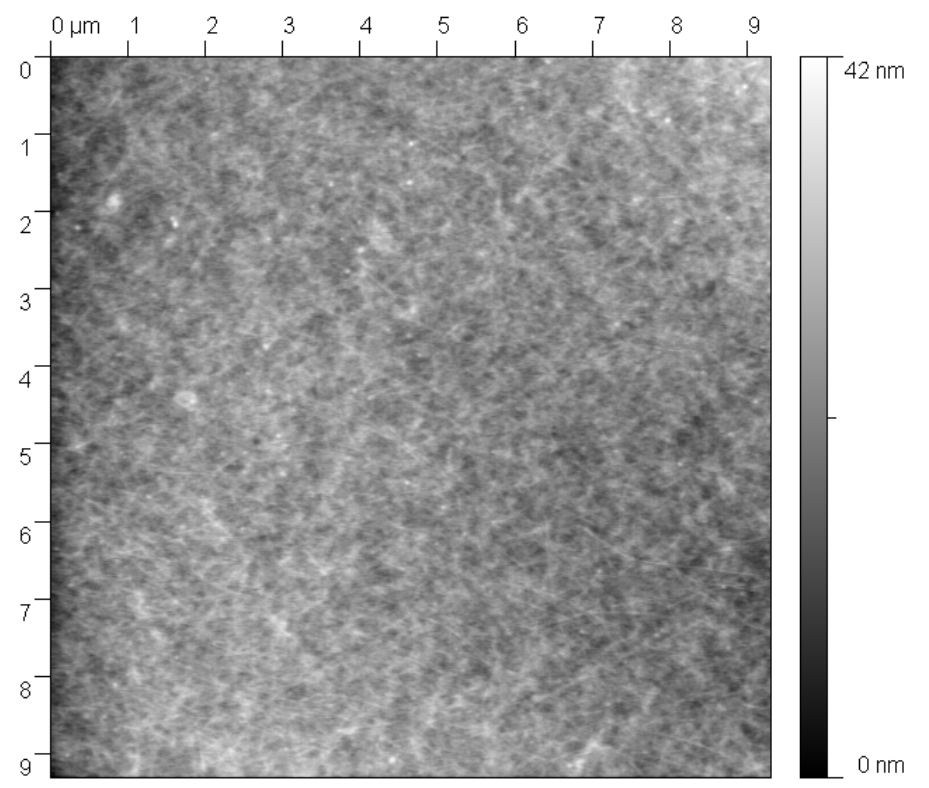

- Sprayed films are more uniform

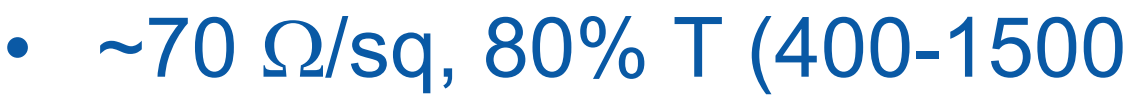
$\mathrm{nm})$

- Smooth and featureless

- Potential for fewer shorts in devices

- Fewer apparent impurities 


\section{Compare TCOs with SWCNTs}

- Transparent SWCNT

- Spaghetti-like network

- $50 \mathrm{~nm}$ thick

- Hole conducting
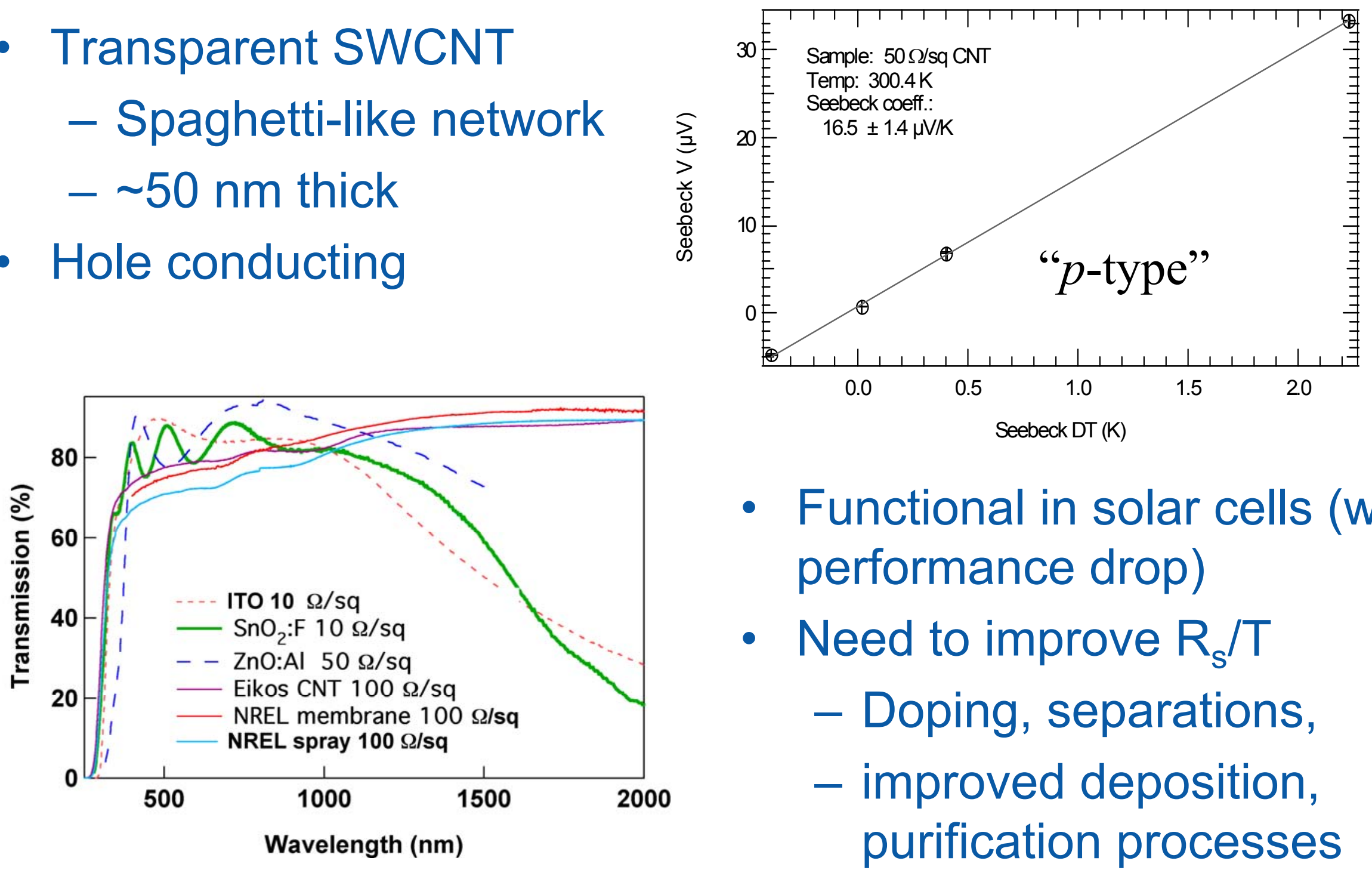

- Functional in solar cells (w/ performance drop)

- Need to improve $R_{s} / T$

- Doping, separations,

- improved deposition, purification processes 


\section{Conductivity in "Bulk" Networks}

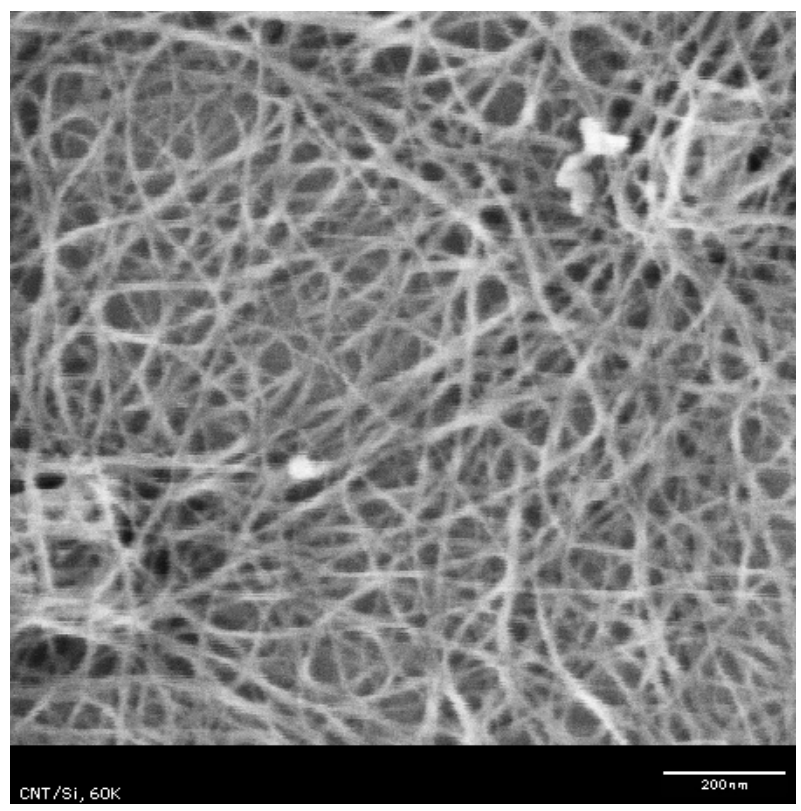

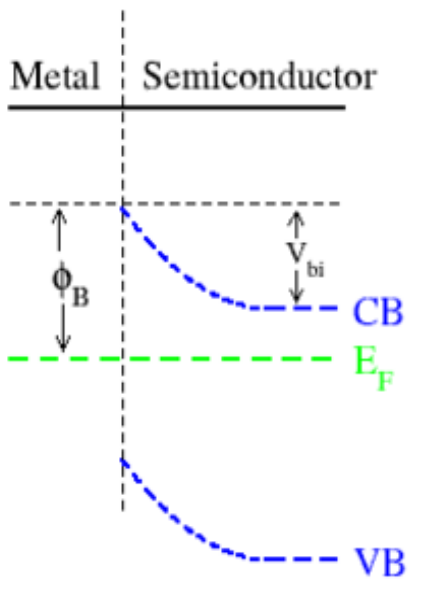

Contact
Schottky barriers are thought to exist between $\mathrm{M}$ and $S$ tubes and limit $\mu$

- Mixture of metallic (1/3) and semiconducting (2/3) tubes

- Tubes/bundles have high aspect ratio in "good" networks

- Measured "p" is low, but networks behave like degenerate semi

- Mobility of the network is very different from individual tubes

$\mu_{\mathrm{H}, \text { tube }} \sim 1000-10000 \mathrm{~cm}^{2} / \mathrm{V}$-s

$\mu_{\mathrm{H}, \text { Network }} \sim 0.02-0.1 \mathrm{~cm}^{2} / \mathrm{V}$-s

Junctions between tubes and bundles dominate conductivity 


\section{SWCNT Network Conductivity}

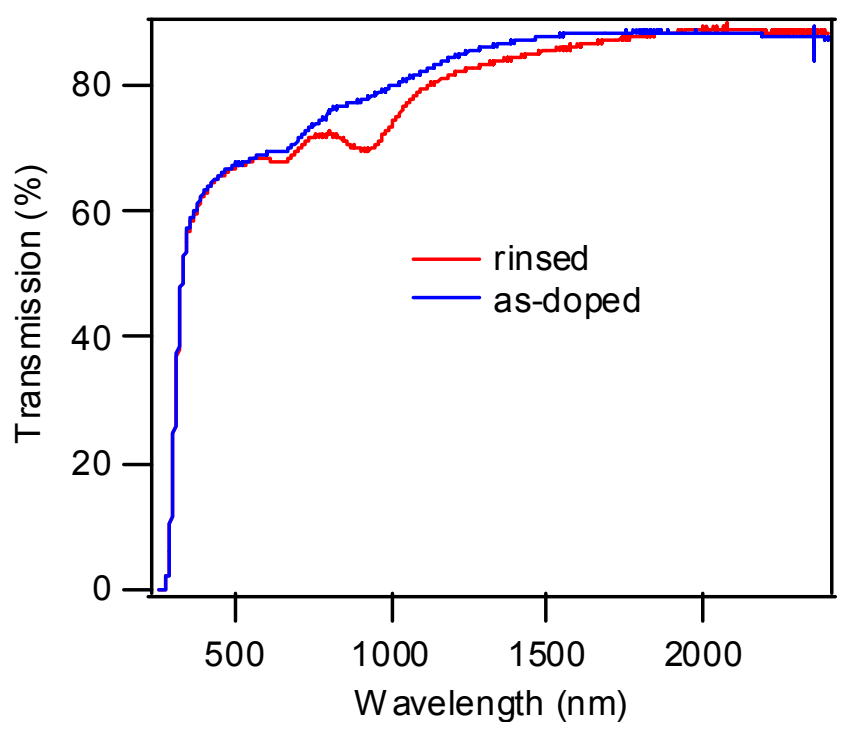

Absorption peaks at 650 and 950 are due to fundamental excitonic transitions of SWCNT
- Networks are soaked in $\mathrm{HNO}_{3}$ after deposition

- Network consolidation

- Conductivity Improvement

$-\mathrm{R}_{\mathrm{s}}=60 \Omega / \mathrm{sq}$

- Rinsing after acid

- Promotes adhesion

- Improves stability

- $R_{\mathrm{s}}$ increases to 108 $\Omega / s q$ 


\section{Devices on NREL SWCNT Networks}

- Ultrasonic spray deposition

- Several 3\% devices

- Thick active layers - spun at 200 rpm

- Reducing electrode roughness is key

- PEDOT can be eliminated
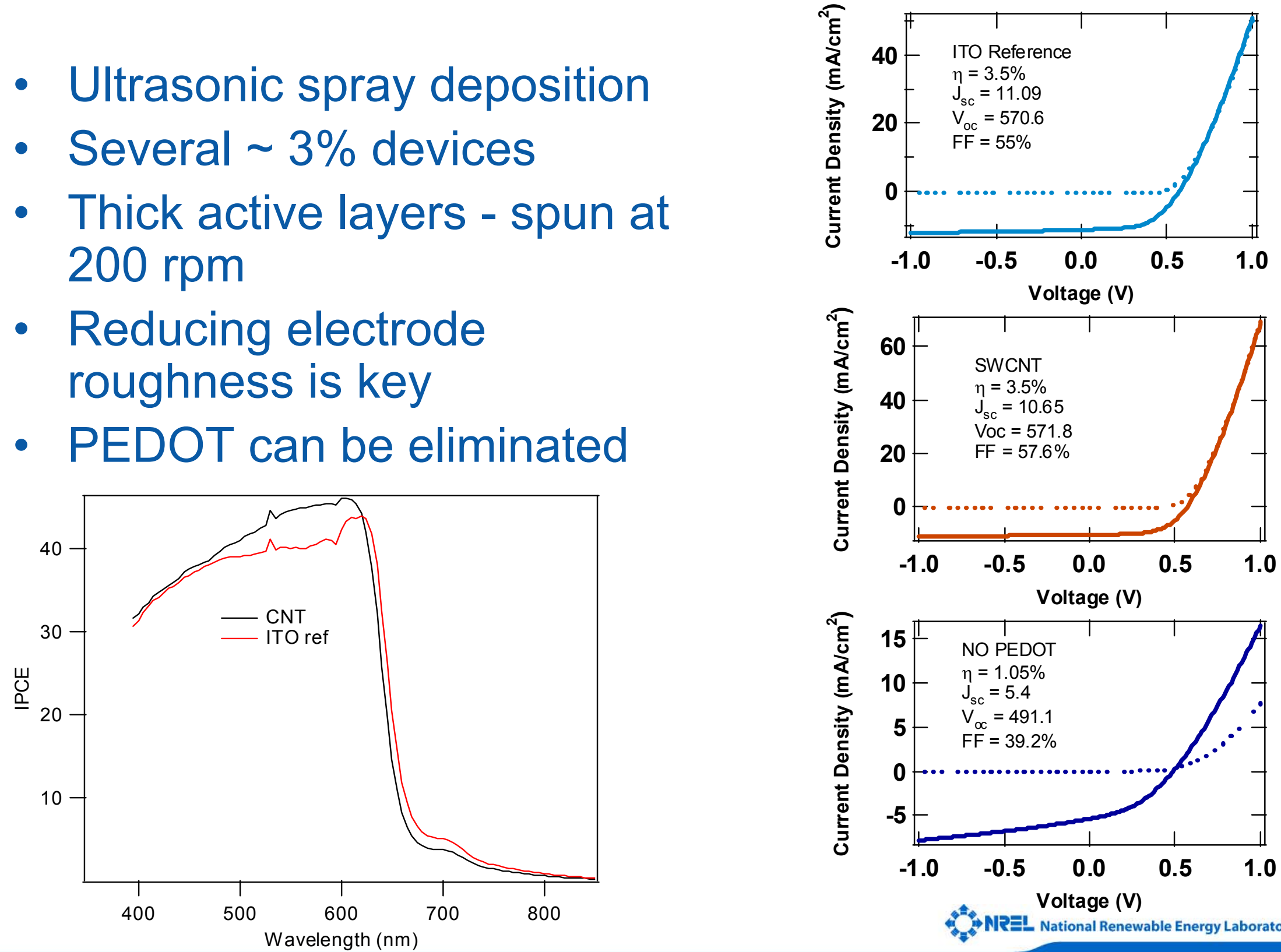


\section{Conclusions}

- SWCNT networks are the best hole-conducting TC available

- Enables new device structures using "p" TC

- SWCNT is a good flexible TC

- Optoelectronic performance is improving

- SWCNT processing, deposition, doping, $\mathrm{m} / \mathrm{s}$ ratio

- Improve process compatibility for better device performance, new architectures (no PEDOT, invert)

- Device performance likely to improve for sprayed or printed active layers

- SWCNT networks could be a model for new TCs

- If a completely black material works as a TC, then what else could we use?? 


\section{Acknowledgements}

- Jao van de Lagemaat, Nikos Kopidakis, Garry Rumbles, Glenn Teeter, Bobby To, Tim Gessert, Joel Duenow, Matt Reese, Xerxes Steirer, Matt White, and many others at NREL

- This work was supported by the U.S. Department of Energy under Contract No. DE-AC36-99GO10337 with the National Renewable Energy Laboratory. funding 